\title{
BOUNDARY VALUE PROBLEMS FOR FIRST-ORDER DIFFERENTIAL EQUATIONS
}

\author{
L. E. BOBISUD AND D. O'REGAN
}

\begin{abstract}
Conditions sufficient to guarantee existence and uniqueness of solutions to multipoint boundary value problems for the first-order differential equation $y^{\prime}=h(t, y)$ are given when $h$ fails to be Lipschitz along a solution of $y^{\prime}=h(t, y)$ and the initial-value problem thus has nonunique solutions.
\end{abstract}

It is well known that the initial value problem for the first-order differential equation $y^{\prime}=h(t, y)$ does not generally have a unique solution if $h$ fails to be Lipschitz in $y$. This raises the possibility, for non-Lipschitz $h$, of well-posedness of problems that would be overspecified if $h$ satisfied a Lipschitz condition; in particular, of the reasonableness of problems that would normally be associated with higher-order equations $[\mathbf{1}, \mathbf{3}]$. Here we examine existence and uniqueness of solutions to two- and multi-point boundary value problems when $y^{\prime}=h(t, y)$ has a solution $y=a(t)$ along which $h$ fails to be Lipschitz. Making the change of variable $y-a(t) \rightarrow y$, we may without loss of generality assume that $h$ vanishes when $y=0$ and that $h$ is not Lipschitz in any neighborhood of $y=0$. We treat first the case of separable variables $h(t, y)=g(t) f(y)$ and then use a comparison theorem to treat the more general case.

Let $b>0$ and consider the boundary value problem

$$
y^{\prime}=g(t) f(y), \quad y(0)=-A, \quad y(b)=B
$$

when $A>0, B>0, g>0$, and $y f(y)<0$ for $y \neq 0$. Then $y^{\prime}<0$ for $y>0$ and $y^{\prime}>0$ for $y<0$, from which it is clear that the problem has no solution. Similarly there is no solution if $y f(y)>0$ for $y \neq 0$. Thus if solutions are to exist in general, then the right-hand side cannot change sign.

It is interesting to note that, since the two-point boundary value problem

$$
y^{\prime \prime}=\lambda y^{1 / 2}, \quad y(0)=y(b)=A>0
$$

has a unique nonnegative solution for each $b>0$ and this solution vanishes on an interval when $\lambda$ is sufficiently large $[\mathbf{2}]$, the following considerations do not extend to higher-order equations.

THEOREM 1. Let $A, B \geq 0$ and let $f \geq 0$ be continuous on $[-A, B]$ and vanish on $(-A, B)$ precisely at $\alpha_{1}<\alpha_{2}<\cdots<\alpha_{k+1}=0<\cdots<\alpha_{n}$. Let $g>0$ be continuous on $[0, b]$. Then there is no solution of the two-point boundary value problem (1) unless the improper integrai

$$
\int_{-A}^{B} \frac{d \zeta}{f(\zeta)}
$$

Received by the editors January 23, 1986.

1980 Mathematics Subject Classification (1985 Revision). Primary 34B15, 34B10. $0002-9939 / 87 \$ 1.00+\$ .25$ per page 
exists. If this integral converges, (1) has continuously differentiable solutions if and only if there exist $T_{1}$ and $T_{2}$ in $[0, b]$ satisfying

$$
\int_{0}^{T_{1}} g(s) d s=\int_{-A}^{0} \frac{d \zeta}{f(\zeta)}, \quad \int_{T_{2}}^{b} g(s) d s=\int_{0}^{B} \frac{d \zeta}{f(\zeta)}
$$

and

$$
T_{1} \leq T_{2}
$$

PROOF. We show first the necessity of these conditions. Suppose $y(t)$ is a solution of (1) and set $\alpha_{0}=-A, \alpha_{n+1}=B(f$ may or may not vanish at these points). On each $\left(\alpha_{i}, \alpha_{i+1}\right), f \neq 0$. Define

$$
\begin{aligned}
t_{i} & =\sup \left\{t: y(t)<\alpha_{i}\right\}, & & i=1,2, \ldots, n+1, \\
s_{i} & =\inf \left\{t: y(t)>\alpha_{i}\right\}, & & i=0,1, \ldots, n .
\end{aligned}
$$

Then $f(y(t)) \neq 0$ on $\left(s_{i}, t_{i+1}\right)$. Let $\varepsilon, \delta>0$ be sufficiently small. Dividing the differential equation by $f(y(t))$, integrating from $s_{i}+\varepsilon$ to $t_{i+1}-\delta$, and passing to the limit as $\varepsilon, \delta \rightarrow 0$ yields

$$
\int_{\alpha_{i}}^{\alpha_{i+1}} \frac{d \zeta}{f(\zeta)}=\int_{y\left(s_{i}\right)}^{y\left(t_{i+1}\right)} \frac{y^{\prime}(t) d t}{f(y(t))}=\int_{s_{i}}^{t_{i+1}} g(t) d t, \quad i=0, \ldots, n .
$$

Thus the improper integral (2) converges. It also follows that

$$
\int_{-A}^{0} \frac{d \zeta}{f(\zeta)}=\sum_{i=0}^{k} \int_{s_{i}}^{t_{i+1}} g(t) d t \leq \int_{0}^{t_{k+1}} g(t) d t
$$

hence there exists a $T_{1} \leq t_{k+1}$ such that the first equality of (3) holds. In the same way, there exists $T_{2} \geq t_{k+1}$ such that the second equality of (3) obtains. The necessity of the hypotheses follows.

To show sufficiency, let $\hat{y}$ be the maximal solution of the initial value problem $y^{\prime}=g(t) f(y), y(0)=-A$; we shall show that $\hat{y}\left(T_{1}\right)=0$. Let $\alpha_{i}, i=0, \ldots, n+1$, be as before; then convergence of the improper integral (2) guarantees the existence of the improper integrals

$$
\int_{\alpha_{i}}^{\alpha_{i+1}} \frac{d \zeta}{f(\zeta)}, \quad i=0, \ldots, n .
$$

Since $T_{1}$ satisfying (3) exists, there also exist $t_{1}<t_{2}<\cdots<t_{k+1} \leq T_{1}$ such that

$$
\sum_{i=1}^{j} \int_{\alpha_{i-1}}^{\alpha_{i}} \frac{d \zeta}{f(\zeta)}=\int_{0}^{t_{j}} g(s) d s \quad(j=1, \ldots, k+1)
$$

set $t_{0}=0$.

Let $y_{i}(i=0, \ldots, k)$ be the maximal solution of the initial value problem

$$
y_{i}^{\prime}(t)=g(t) f\left(y_{i}(t)\right), \quad y_{i}\left(t_{i}\right)=\alpha_{i} ;
$$

we shall show first that $y_{i}$ is defined on $\left[t_{i}, t_{i+1}\right]$ and $y_{i}\left(t_{i+1}\right)=\alpha_{i+1}$. To this end let $y_{i, \varepsilon}$, for all sufficiently small $\varepsilon>0$, be the maximal solution of

$$
y_{i, \varepsilon}^{\prime}=g(t) f\left(y_{i, \varepsilon}\right)+\varepsilon, \quad y_{i, \varepsilon}\left(t_{i}\right)=\alpha_{i}+\varepsilon
$$


If $y_{i}$ is defined on $\left[t_{i}, s_{i}\right)$, then for any $\delta>0, y_{i, \varepsilon}$ exists for $t_{i} \leq t \leq s_{i}-\delta$ for all sufficiently small $\varepsilon$, and $y_{i, \varepsilon} \downarrow y_{i}$ as $\varepsilon \downarrow 0$ [4]. Suppose that $y_{i}(t) \equiv \alpha_{i}$ on $\left[t_{i}, \bar{t}_{i}\right]$ with $\bar{t}_{i}>t_{i}$ and $\bar{t}_{i}$ small enough that $\theta$ defined by

$$
\int_{\alpha_{i}}^{\theta} \frac{d \zeta}{f(\varsigma)}=\int_{\bar{t}}^{\bar{t}_{i}} g(t) d t
$$

exists and satisfies $\theta<\alpha_{i+1}$; this is guaranteed by the existence of (2). Since $g>0$, $\theta>\alpha_{i}$. For $\varepsilon_{0}>0$ sufficiently small, the maximal solution $y_{i, \varepsilon}$ of (5) exists on $\left[t_{i}, \bar{t}_{i}\right]$ for $0<\varepsilon \leq \varepsilon_{0}$. By further reducing $\bar{t}_{i}$ and $\theta$, if necessary, we may assume that $y_{i, \varepsilon}(t)<\alpha_{i+1}$ for $0<\varepsilon \leq \varepsilon_{0}$ and $t \in\left[t_{i}, \bar{t}_{i}\right]$. Then $\alpha_{i}<y_{i, \varepsilon}(t)<\alpha_{i+1}$ on $\left(t_{i}, \bar{t}_{i}\right)$, so $f\left(y_{i, \varepsilon}(t)\right)>0$ there, and we get from (5) that

$$
\int_{t_{i}}^{\bar{t}_{i}} \frac{y_{i, \varepsilon}^{\prime}(t) d t}{f\left(y_{i, \varepsilon}(t)\right)} d t>\int_{t_{i}}^{\bar{t}_{i}} g(t) d t
$$

and hence that

$$
\int_{\alpha_{i}}^{y_{i, \varepsilon}\left(\bar{t}_{i}\right)} \frac{d \zeta}{f(\zeta)}>\int_{\alpha_{i}+\varepsilon}^{y_{i, \varepsilon}\left(\bar{t}_{i}\right)} \frac{d \zeta}{f(\zeta)}>\int_{t_{i}}^{\bar{t}_{i}} g(t) d t .
$$

Thus $y_{i, \varepsilon}\left(\bar{t}_{i}\right)>\theta$ for $0<\varepsilon \leq \varepsilon_{0}$, whence $y_{i}\left(\bar{t}_{i}\right)=\lim _{\varepsilon \rightarrow 0} y_{i, \varepsilon}\left(\bar{t}_{i}\right) \geq \theta>\alpha_{i}$, a contradiction. We have therefore shown that $y_{i}(t)=\alpha_{i}$ only for $t=t_{i}$.

Suppose now that $\alpha_{i}<y_{i}(t)<\alpha_{i+1}$ for $t_{i}<t<s$. Then $f\left(y_{i}(t)\right)>0$, so we get that

$$
\int_{y_{i}\left(t_{i}+\varepsilon\right)}^{y_{i}(s-\delta)} \frac{d \zeta}{f(\zeta)}=\int_{t_{i}+\varepsilon}^{s-\delta} g(t) d t
$$

for sufficiently small $\varepsilon, \delta>0$. Passing to the limit as $\varepsilon \rightarrow 0, \delta \rightarrow 0$, we have that

$$
\int_{\alpha_{i}}^{y_{i}(s-)} \frac{d \zeta}{f(\zeta)}=\int_{t_{i}}^{s} g(t) d t
$$

But we have

$$
\int_{\alpha_{i}}^{\alpha_{i+1}} \frac{d \zeta}{f(\zeta)}=\int_{t_{i}}^{t_{i+1}} g(t) d t
$$

Therefore if $s<t_{i+1}$, then $y_{i}(s-)<\alpha_{i+1}$ and $y_{i}$ can be extended to the right of $s$. So $s \geq t_{i+1}$. But the unique solution to

$$
\int_{\alpha_{i}}^{\theta} \frac{d \zeta}{f(\varsigma)}=\int_{t_{i}}^{t_{i+1}} g(s) d s
$$

is $\theta=\alpha_{i+1}$. Therefore $y_{i}\left(t_{i+1}\right)=\alpha_{i+1}$.

We have shown that on each interval $\left[\alpha_{i}, \alpha_{i+1}\right]$ the maximal solution $y_{i}$ of $y_{i}^{\prime}=$ $g(t) f\left(y_{i}\right), y_{i}\left(t_{i}\right)=\alpha_{i}$ exists and satisfies $y_{i}\left(t_{i+1}\right)=\alpha_{i+1}$. Since $f\left(\alpha_{i}\right)=0$ for $i=1, \ldots, k$, we have easily that $y_{i}^{\prime}\left(\alpha_{i}-\right)=y_{i}^{\prime}\left(\alpha_{i+1}+\right)=0(i=1, \ldots, k)$ and $y_{0}^{\prime}\left(\alpha_{1}-\right)=0$. It follows that $\hat{y}_{1}$ defined by

$$
\hat{y}_{1}(t)=y_{i}(t), \quad t_{i} \leq t<t_{i+1},
$$

is the maximal solution of $y^{\prime}=g(t) f(y), y(0)=-A$ on $\left[0, T_{1}\right)$ and $\hat{y}_{1}\left(T_{1}-\right)=$ $\hat{y}_{1}^{\prime}\left(T_{1}-\right)=0$. 
In the same way we conclude there exists the minimal solution of

$$
\hat{y}_{2}^{\prime}(t)=g(t) f\left(\hat{y}_{2}(t)\right), \quad \hat{y}_{2}(b)=B
$$

defined on $\left(T_{2}, b\right]$ and satisfying $\hat{y}_{2}\left(T_{2}+\right)=\hat{y}_{2}^{\prime}\left(T_{2}+\right)=0$. Therefore, since $T_{1} \leq T_{2}$,

$$
y(t) \equiv \begin{cases}\hat{y}_{1}(t), & 0 \leq t<T_{1}, \\ 0, & T_{1} \leq t \leq T_{2}, \\ \hat{y}_{2}(t), & T_{2}<t \leq b,\end{cases}
$$

defines a classical solution of (1), proving the theorem.

COROLLARY 1. If $f$ and $g$ are as in the theorem and $\int_{0}^{\infty} g=\int_{-\infty}^{0} g=\infty$, then the two-point boundary problem (1) has a solution for each $A, B \geq 0$ provided $b$ is sufficiently large (depending on $A$ and $B$ ).

The following result is an easy extension to the multi-point boundary value problem. It is interesting to compare it with corresponding results for higher-order Lipschitz equations [3].

COROLLARY 2. Let $f$ and $g$ satisfy the hypotheses of the theorem. Let $C_{i} \in$ $\left(\alpha_{i}, \alpha_{i+1}\right)$ for $i=1, \ldots, n-1$ and $0<t_{1}<\cdots<t_{n-1}<b$ be given. Then the boundary value problem

$$
y^{\prime}=g(t) f(y), \quad y(0)=-A, \quad y\left(t_{1}\right)=C_{1}, \ldots, y\left(t_{n-1}\right)=C_{n-1}, \quad y(b)=B
$$

has a solution if and only if there exist sequences $\left\{T_{i}\right\}$ and $\left\{S_{i}\right\}$ such that

$$
0<T_{1} \leq S_{1}<t_{1}<T_{2} \leq S_{2}<t_{2}<\cdots \leq S_{n}<b
$$

and

$$
\begin{gathered}
\int_{-A}^{\alpha_{1}} \frac{d \zeta}{f(\zeta)}=\int_{0}^{T_{1}} g(t) d t, \quad \int_{\alpha_{i}}^{C_{i}} \frac{d \zeta}{f(\zeta)}=\int_{S_{i}}^{t_{i}} g(t) d t, \\
\int_{C_{i}}^{\alpha_{i+1}} \frac{d \zeta}{f(\zeta)}=\int_{t_{i}}^{T_{i+1}} g(t) d t \quad(i=1, \ldots, n-1), \\
\int_{\alpha_{n}}^{B} \frac{d \zeta}{f(\zeta)}=\int_{S_{n}}^{b} g(t) d t .
\end{gathered}
$$

The following theorem establishes conditions under which the two-point boundary value problem has a unique solution. Extension to the multi-point problem is straightforward and will be omitted.

THEOREM 2. Let the hypotheses of Theorem 1 hold and, in addition, suppose that $f$ vanishes on $[-A, B]$ only at zero and that $f$ is locally Lipschitz on $[-A, 0) \cup$ $(0, B]$. Then the solution of the two-point boundary value problem (1) is unique.

PROOF. Since $f$ is locally Lipschitz, the standard existence-uniqueness theorem forces uniqueness of the solutions $\hat{y}_{1}$ and $\hat{y}_{2}$ constructed in the proof of Theorem 1; $T_{1}$ and $T_{2}$ are also unique. Since any solution of the differential equation is nondecreasing, the solution $y(t)$ of the boundary value problem given by $(6)$ is now seen to be unique.

EXAMPLE. Let $g(t) \equiv 1, f(y) \equiv|y|^{\alpha}$. Then (1) has no solution unless $0<\alpha<1$. For $0<\alpha<1$, the two-point boundary value problem (1) has a solution, which is unique, if and only if $(1-\alpha) b \geq A^{1-\alpha}+B^{1-\alpha}$. 
We now turn to existence of solutions of the two-point boundary value problem for $y^{\prime}=h(t, y)$; this will be established by means of a comparison theorem. A similar result for the multi-point problem is easily established along the same lines.

The following lemma is well known; a proof may be found in [4].

LEMMA. Let $g$ be continuous on $E$, an open set in $\mathbf{R}^{2}$, and let the maximal solution $u(t)$ of

$$
u^{\prime}=g(t, u), \quad u\left(t_{0}\right)=u_{0}
$$

exist on $\left[t_{0}, t_{0}+a\right)$. Let $v(t)$ be continuous on $\left[t_{0}, t_{0}+a\right)$ with $(t, v(t)) \in E$ for $t \in\left[t_{0}, t_{0}+a\right)$, and suppose that

$$
v^{\prime}(t) \leq g(t, v(t)), \quad v\left(t_{0}\right) \leq u_{0} \quad\left(t_{0}<t<t_{0}+a\right)
$$

Then

$$
v(t) \leq u(t) \text { for } t_{0} \leq t<t+a .
$$

Let the minimal solution $w(t)$ of $(7)$ exist on $\left(t_{0}-a, t_{0}\right]$. Let $v(t)$ be continuous on $\left(t_{0}-a, t_{0}\right]$ with $(t, v(t)) \in E$ for $t \in\left(t_{0}-a, t_{0}\right]$, and suppose that

$$
v^{\prime}(t) \leq g(t, v(t)), \quad v\left(t_{0}\right) \geq u_{0} \quad\left(t_{0}-a<t<t_{0}\right) .
$$

Then

$$
v(t) \geq w(t) \quad \text { for } t_{0}-a<t \leq t_{0} .
$$

With the aid of this result, we can easily prove the following comparison theorem.

THEOREM 3. Let the two-point boundary value problem

$$
z^{\prime}=\tilde{h}(t, z), \quad z(0)=-\tilde{A}, \quad z(b)=\tilde{B},
$$

where $\tilde{A} \geq 0, \tilde{B} \geq 0$, possess a solution $z(t)$, not necessarily unique. Suppose that

(i) $0 \leq A \leq \tilde{A}, 0 \leq B \leq \tilde{B}$,

(ii) $h$ is continuous on an open set $E$ containing $[0, b] \times[-\tilde{A}, \tilde{B}]$,

(iii) $h(t, 0) \equiv 0$ for $t \in[0, b]$,

(iv) $h(t, u) \geq \tilde{h}(t, u)$ on $E$.

Then the boundary value problem

$$
y^{\prime}=h(t, y), \quad y(0)=-A, \quad y(b)=B
$$

has a solution.

ProOF. Let $y_{1}$ be the maximal solution of the initial value problem

$$
y^{\prime}=h(t, y), \quad y(0)=-A \text {. }
$$

Since $z$ solves

$$
z(0)=-\tilde{A} \leq-A, \quad z^{\prime}(t)=\tilde{h}(t, z(t)) \leq h(t, z(t)),
$$

we have from the first part of the Lemma that $z(t) \leq y_{1}(t)$ as far to the right of zero as $y_{1}$ exists. Let

$$
t_{1}=\min \{t \in[0, b]: z(t)=0\} .
$$

Since the maximal solution $y_{1}$ can be extended until it leaves the set $[0, b] \times[-A, B]$, there must exist $T_{1} \leq t_{1}$ such that $y_{1}\left(T_{1}\right)=0$. From the differential equation (8) we get that $y_{1}^{\prime}\left(T_{1}-\right)=0$. 
Let $y_{2}(t)$ be the minimal solution of the terminal value problem

$$
y_{2}^{\prime}=h\left(t, y_{2}\right), \quad y_{2}(b)=B
$$

and let

$$
t_{2}=\max \{t \in[0, b]: z(t)=0\} .
$$

Necessarily $t_{2} \geq t_{1}$. By the second part of the Lemma we have that $y_{2}(t) \leq z(t)$ as far to the left of $b$ as $y_{2}$ exists; as before it follows that there is a $T_{2} \geq t_{2}$ such that $y_{2}$ is defined on $\left[T_{2}, b\right]$ and $y_{2}\left(T_{2}\right)=y_{2}^{\prime}\left(T_{2}+\right)=0$. Since $T_{2} \geq T_{1}$, it follows that

$$
y(t) \equiv \begin{cases}y_{1}(t), & 0 \leq t<T_{1}, \\ 0, & T_{1} \leq t \leq T_{2}, \\ y_{2}(t), & T_{2}<t \leq b,\end{cases}
$$

is a classical solution of the two-point boundary value problem (8).

This comparison theorem can be used both to prove existence and to prove nonexistence of solutions of the two-point boundary value problem. As an example of the former, the following result is immediate.

Corollary. Let $f, g, A, B, b$ satisfy the hypotheses of Theorem 1, and let

$$
h(t, y) \geq g(t) f(y),
$$

where $h$ also satisfies hypotheses (ii)-(iii) of Theorem 3. Then the boundary value problem (8) has at least one solution.

THEOREM 4. Let $A, B>0$; let $h(t, y) \geq 0$ for $(t, y) \in[0, b] \times[-A, B]$ and vanish precisely when $y=0$; let $h$ be continuous on $[0, b] \times[-A, B]$ and locally Lipschitz on $[0, b] \times([-A, 0) \cup(0, B])$. Then the two-point boundary value problem

$$
y^{\prime}=h(t, y), \quad y(0)=-A, \quad y(b)=B
$$

cannot have two distinct solutions.

The proof does not differ materially from that of Theorem 2 and so will be omitted, as will the extension to multi-point problems.

REMARK. The simple technique employed here can be applied readily to other forms of boundary conditions; for example, to the integral conditions imposed in $[\mathbf{5}]$.

\section{REFERENCES}

1. P. Bailey, L. Shampine and P. Waltman, Nonlinear two point boundary value problems, Academic Press, New York, 1968.

2. C. Bandle, R. P. Sperb and I. Stakgold, Diffusion and reaction with monotone kinetics, Nonlinear Anal. 8 (1984), 321-333.

3. L. K. Jackson, Existence and uniqueness of solutions of boundary value problems for Lipschitz equations, J. Differential Equations 32 (1979), 76-90.

4. V. Lakshmikantham and S. Leela, Differential and integral inequalities: Theory and applications, vol. 1, Academic Press, New York, 1969.

5. A. Yoshikawa, On a simple singular perturbation problem, J. Differential Equations 60 (1985), $363-380$.

Department of Mathematics and Applied Statistics, University of Idaho, MOSCOW, IDAHO 83843 